\title{
D-ZERO SILICON COOLING SYSTEM DESCRIPTION
}

D-ZERO ENGINEERING NOTE 3823.112-EN-550

Author: Herman Cease, PPD/ETT/D-Zero Mech.

February 9, 2001

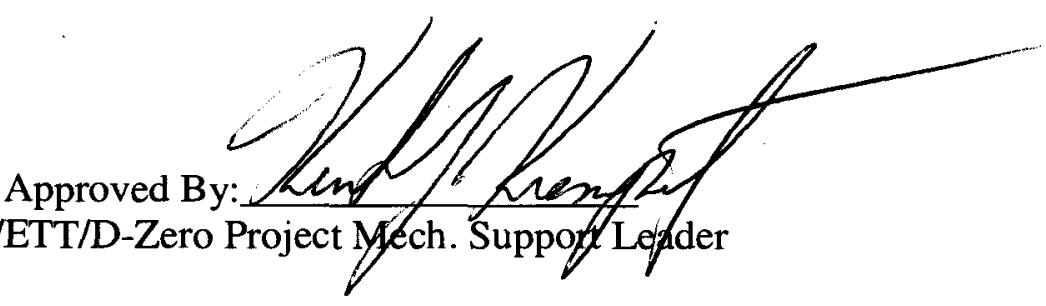




\section{D-Zero Silicon Cooling System}

Update 2

Bruce Squires ('98)/Herman Cease

January 05, 2001

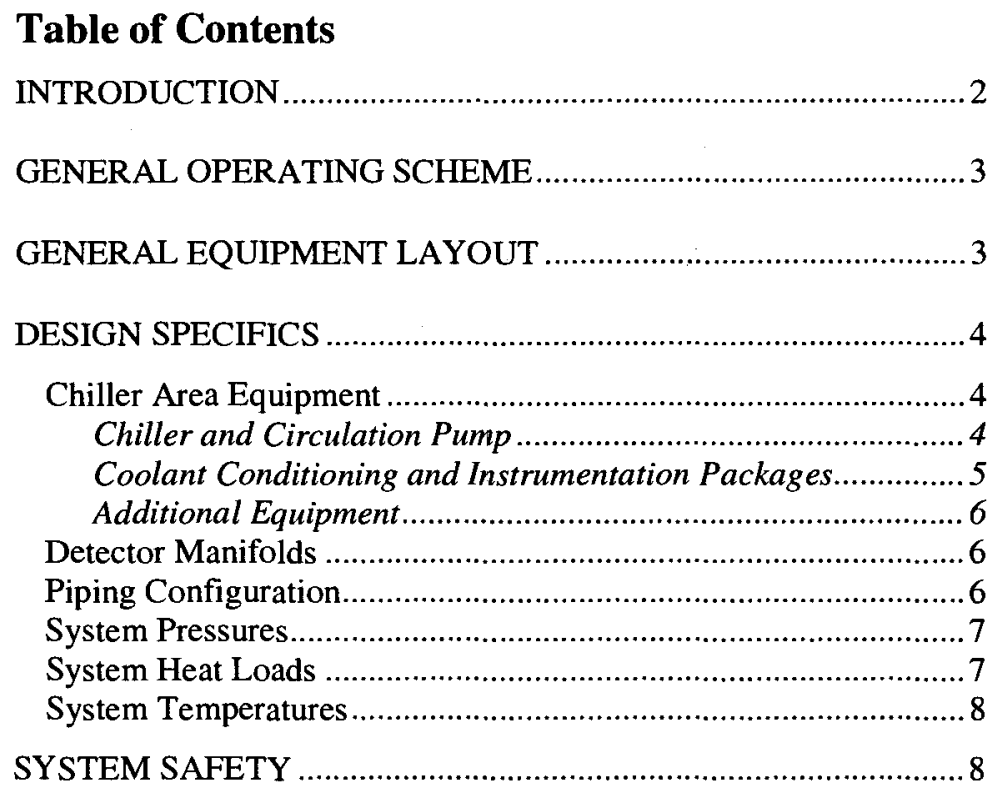

\section{INTRODUCTION}

The silicon detector is cooled by means of a coolant in the beryllium channels that also act as the primary supporting device for the silicon ladders and wedges. The coolant is water with ethylene glycol added as a freezing point depressant. The glycol concentration in the coolant is $30 \%$ by weight resulting in a freezing point of approximately $-15^{\circ} \mathrm{C}$. If the water/glycol is not sufficient for maintaining the desired detector temperature, the concentration of the water/glycol may be changed or an alternative coolant may be used. 


\section{GENERAL OPERATING SCHEME}

The silicon detector is cooled by a closed loop cooling system. The pressure in the detector cooling channels is maintained below atmospheric pressure. This is obtained by locating an atmospheric expansion upstream and with an elevation slightly lower than the detector. This expansion tank is purged with dry nitrogen to prevent condensation and to prevent oxygen from absorbing into the coolant. The coolant circulation pump is located downstream from the detector so that it will further reduce the pressure in the cooling channels when the pump is operating.

The purpose behind maintaining the cooling channels below atmospheric pressure is to ensure that leaks in the cooling system will not cause the coolant to drip or squirt out of the system onto the detectors. Instead, this will result in air/nitrogen entering the cooling system. Air separators are located downstream of the detector.

The temperature of the coolant is maintained constant for all detector operating conditions. The coolant is distributed to the cooling channels from manifolds. Since each cooling channel is sized to have similar pressure drop for the nominal flow, no balancing valves are required. This requires that the manifolds are designed to minimize mass while not starving any of the cooling channels. These two aspects of the design simplify the controls of the cooling system. The system is cooled by reducing the setpoint of the controller in the chiller and the overall flow rate is manually set to its appropriate value. From this point on, the system needs to be monitored to ensure that everything is operating properly. Leaks can be monitored by watching the level in the expansion tank, the overall flow rate and pressures within the system.

\section{GENERAL EQUIPMENT LAYOUT}

The location for the chillers is on the south sidewalk of the D-Zero Assembly Building. The piping for the detector will service both the Collision and Assembly Hall locations. Most of the piping is vacuum insulated where practicable. The area under the edge of the sidewalk where the calorimeter piping runs between the two halls is used for part of the path. The pipes are connected to the platform in the cryocorner area on the platform.

The location for the expansion tank is on the east side of the cryo-bridge at the same elevation as the detector. A fire-hose is attached to the top of the tank and runs down to the pit floor. The hose is used as a N2 gas vent and as a spill trough in the event the expansion tank overflows.

The manifolds for the detector are designed to have the manifold run the entire length of the detector. The manifolds were constructed from PVC pipe. On each side of the detector is a supply and return manifold. The supply manifold is the lower set of manifolds. The mass of the manifolds is minimized by reducing the diameter of the supply manifolds as flow is taken out for the cooling channels and increasing the return manifold diameter when adding flow from a cooling channel.

The piping outside the area of the CFT is vacuum jacketed stainless steel. The transition from plastic to metal piping is done near barrel 3 of the CFT and is done with hose and hose barb type fittings. 
The location for the air separator tank is on the south sidewalk upstream from the chiller. A second tank is located above the crane rail near the ceiling of the building. This tank acts as an elevated dry volume to ensure coolant is not carried into the vacuum pumps.

\section{DESIGN SPECIFICS}

In this section, the current details of the design are laid out. There are many features being designed into the cooling system to enhance the inspection, operation and coolant quality. Whenever possible, equipment is located near the chillers in the assembly hall in order to provide ease of access and reduce the need for accesses to the collision hall.

The nominal system flow rate for the system is about $40 \mathrm{lpm}$. This assumes an 8 psi pressure drop over the cooling channels. The coolant temperature is $-10^{\circ} \mathrm{C}$.

All process transmitters in the system will be connected to the industrial controls system at DAB. A parallel electrical signal is also passed to the EPICS data system.

\section{Chiller Area Equipment}

The area around the chillers contain most of the instrumentation and coolant conditioning equipment. Immediately downstream of the chiller pump is the coolant conditioning package. This is where coolant is filtered and de-ionized. Downstream of this package is the instrumentation package where the coolant properties are monitored and the flow rate is measured.

\section{Chiller and Circulation Pump}

The chiller is made by Applied Process Equipment Corp and is a modified version of their model AC3. The cooling capacity of the unit is between 4.5 and $6.6 \mathrm{~kW}$. The circulation pump is located within the chiller unit. It is a centrifugal pump with a head of about $35 \mathrm{psid}$ for $40 \mathrm{lpm}$ of $-10^{\circ} \mathrm{C}$ water/ethylene glycol $(30 \% \mathrm{w})$. Since the pump is centrifugal, the head pressure is controlled by controlling the flow through the pump. This is performed by a manual bypass valve that goes between the chiller supply and return piping. Additional valves on the supply and return piping are installed for additional pressure control if necessary. These valves mainly act as a way to isolate the chiller when changing out the chiller for the spare chiller. The chiller's piping connections are 1" pipe on the supply side and 2 " pipe on the return side. The supply piping in the chiller area is 1" pipe. It is not a pressure drop issue. The return piping in this area is $1-1 / 2$ ".

The spare chiller is installed in parallel with the primary chiller. Both chillers should not be operated at the same time. If this pressure drops below the Net Positive Suction Head required (NPSHr), the pump will cavitate and will be destroyed.

Pressure instrumentation is installed on the supply and return piping of the chiller. This is required to monitor these pressures during start-up and while monitoring operation of the system. Both a pressure transmitter and a dial gauge are installed at these locations. Additionally, the pressure will be monitored in the instrumentation 
package since there may be significant pressure drop between the chiller and the platform.

\section{Coolant Conditioning and Instrumentation Packages}

The coolant needs to be maintained within specified parameters to reduce the potential for corrosion and scaling. The coolant must be monitored for electrical conductivity to prevent the fluid from being a ground path for the detector. Since the glycol concentration has a significant effect on the pressure drop in the system, this is monitored. The coolant conditioning area is located by the chillers and is where all of this monitoring and conditioning will occur.

The properties that are measured are the electrical conductivity, $\mathrm{pH}$ and refractive index. The electrical conductivity measurement is quite straight forward in its application. A de-ionizer will be provided on a small bypass leg of the main flow line. This de-ionizer may be used to reduce the conductivity of the coolant. Since it is a small flow rate, the change when it is turned on will be slow.

The $\mathrm{pH}$ meter which can also monitor the oxidation potential is the probable device for monitoring the corrosiveness of the coolant. How to make the adjustments to the $\mathrm{pH}$ have not been determined at this time. If an inhibitor must be used, we may either have to install a device for monitoring its concentration or we may have to take samples on a periodic basis.

The refractive index will give us a good measure of the glycol concentration of the coolant. Periodic sampling is required. The glycol concentration will only change if we must periodically remove gas from the system due to a leak because the gas will be saturated with moisture. Water can be added by opening a valve on the return side of the pump and slowly sucking the water from a water supply. This valve is provided on the vessel upstream of the chiller pump and air separator tank.

The $\mathrm{pH}$ of the fluid is not the only concern for corrosion and scaling. Bacteria is a common problem in water based cooling systems. An ultraviolet sterilizer was investigated but is not part of the system Union carbide, the supply of the ethylene glycol, indicated that bacteria "prefers not to grow in the coolant when the glycol concentration is above $20 \%$ ". However, glycol is not specifically considered a biocide. As an added precaution, the system will be made light tight wherever possible.

To monitor the corrosiveness of the coolant, an inspection spool piece is installed. This section contains a piece of beryllium that is electrically insolated from the piping. It would be ideal if this piece is at the same potential as the cooling channels. This would give us an accurate visual and physical check on the state of corrosion in the system. The resistance of the beryllium piece can be periodically monitored for changes which are inevitable with corrosion. A sight glass is installed to view the beryllium piece. A light blocking cover will be removed to view the piece. The spool piece can also be bypassed for removal and closer inspection.

For added protection of the components, filters are installed in the main flow line. Filters are alos located before and after the Deionizer tank. A bypass is provided around the filters to facilitate replacing the filter elements when necessary. The filter may only have to be replaced after the first time the system is filled with coolant and circulated for a few hours. It is more likely that the filter will never be changed. A differential pressure gauge is in the design to monitor the resistance of the filter package. As the filters trap 
more particles, the elements will become more resistive and the pressure reading will give the best indication of the time to change the filters. The filter media is made from pleated polypropylene fabric.

The flow rate of the coolant is measured in the instrumentation package using a thermal flow sensor. This style of sensor has the advantage of no moving parts to wear. A mechanical flow meter is also installed in the supply and both return lines.

\section{Additional Equipment}

In order to monitor the flow balancing between the north and south manifolds, flow meters and pressure gauges are installed in each of the two return lines. These two lines will merge near the chiller area. The pressure drop versus flow rate will be characterized for each section in order to get an approximation of the differences between the two piping runs.

\section{Detector Manifolds}

A supply and return line is located at both the east and west sides of the silicon detector. The manifolds are then duplicated for the north and south halves. The supply and return lines are fed from EC/CC crack at the 6 O'clock position of the CC. Flow balancing between the south and north manifolds is accomplished by monitoring the flow rates in the two return lines. Since there is a requirement for minimizing the mass of these devices, the manifolds were stepped down when possible. It is important to maintain the desired flow through each cooling channel.

The connections on the cooling channels are done inside the silicon detector support trough. The nozzles on the silicon bulkheads are actually glued to the cooling channels allowing for easier fabrication as well as flexibility on the direction in which the nozzle can point. It appears that the best connection on the barrel F-disks is at about $15^{\circ}$ away from the beamline and towards the bulkhead that the disk is attached to. The end Fdisks are very tight at $45^{\circ}$. The $\mathrm{H}$-disk nozzles are at a $45^{\circ}$ angle from the disk.

The manifold connections are pointed radially inward. This makes the final connection difficult. This forces the tubing to be placed on the cooling channels first, then fed through the half cylinder (without the manifolds), connecting the tubes to the manifolds and then pushing the tubes back into the half cylinder and mounting the manifolds.

The manifold to bulkhead connections were done with Cilran tubing. The joints were fastened using a double wire wrap using brass wire.

\section{Piping Configuration}

As mentioned earlier, most of the piping in the system will be vacuum insulated. The purpose is to keep the piping heat load lower that the detector heat load. Due to the cooling capacity of the chillers, this is now very desirable if we wish to avoid using both chillers at once. It's mainly the long runs of piping that are insulated in this fashion. The remainder of the valves, instrumentation, vessels and equipment are foam insulated. The 
additional heat load due to using foam in these areas should be modest and will save significant cost and effort.

All of the supply tubing between the chiller area and the expansion tank will be 1 " tubing. Where the supply tubing splits for the south and north manifolds, the tubing reduces to $3 / 4$ " pipe. The return tubing from near the detector to the sidewalk area are $3 / 4$ " pipe. Where these two return lines combine the tubing will increase to 1-1/2" pipe.

\section{System Pressures}

The pressures and pressure drops of most concern are the pressure drop from the expansion tank through the detector to the chiller pump, the absolute pressure at the suction side of the chiller, the pressure difference between the suction side of the chiller pump and the vacuum pressure above the air separator tank, and the overall pressure drop across the chiller pump. The pressure drop from the expansion tank through the detector to the chiller pump must be less than 1 atm to prevent the pump from cavitating. The absolute pressure at the suction side of the chiller must be greater than the evaporation point of the coolant. The pressure difference between the suction side of the chiller pump and the vacuum pressure above the air separator tank has to be greater than the physical head height of the air separator tank. If the differential pressure becomes less, the air separator tank will empty and the coolant will overflow the expansion tank. The overall pressure drop across the chiller pump must meet the flow curve for the chiller pump at the desired flow.

\section{System Heat Loads}

The heat load for this design is assumed to be $640 \mathrm{~mW}$ per SVX2 chip. It is likely that the chip power will be significantly less than this. Maybe closer to $425 \mathrm{~mW}$ per chip. Therefore, this system is being designed for a 3962.88 Watt detector heat load using the $640 \mathrm{~mW}$ value. Table 1 is a summary of each barrel and disk heat load due to the chips. Table 1 lists the heat loads considered for this system. The table is not complete but it does include what are assumed to be the major heat sources. The piping system heat loads total about $0.9 \mathrm{~kW}$ bringing the total system heat load to about $4.9 \mathrm{~kW}$.

The piping heat load is expected to be a few hundred Watts. Most of the piping on the platform and the transfer lines to the platform are vacuum jacketed. The lines on the platform were baked out at $400^{\circ} \mathrm{C}$ to minimize outgassing and Vacuum getter material was added to maintain a pressure less than 20 microns. The transfer lines to the platform have super-insulation in the vacuum to minimize heat losses. The piping on the sidewalk near the chillers and the tanks are foam insulated. The pump inefficiency is the greatest heat load in the piping system. At $10 \mathrm{gpm}$ the estimated effeciency is $15 \%$ and the pump requires 1.15 H.P. The coolant heating due to the pump is about 700 Watts. Again, this does not take into account every item that will be in the system nor the

ambient heat load on the detector. 
Table 1. Barrel and Disk Heat Loads

\begin{tabular}{|c|c|c|}
\hline Inner 4 barrels: & 36 & 6-chip ladders $=216$ chips $=138.24$ watts \\
\hline & 36 & 9-chip ladders $=324$ chips $=207.36$ watts \\
\hline & Tot: & $1=345.60$ watts divided between 2 passages. \\
\hline Outer 2 barrels: & 36 & 3-chip ladders $=108$ chips $=69.12$ watts \\
\hline & 36 & 9-chip ladders $=324$ chips $=207.36$ watts \\
\hline & Tot: & $1=276.48$ watts divided between 2 passages. \\
\hline F-disk: & 12 & 14-chip wedges $=168$ chips $=107.52$ watts. \\
\hline H-disk: & 4 & 6 -chip wedges $=288$ chips $=184.32$ watts. \\
\hline Piping losses & & 300 Watts \\
\hline Pump inefficiency & & 700 watts \\
\hline TOTAL & & $5.0 \mathrm{~kW}$ \\
\hline
\end{tabular}

\section{System Temperatures}

This discussion will assume that the coolant temperature at the cooling channels is $-10^{\circ} \mathrm{C}$. The heat loads for the system are estimated based upon what is believed to be the worst case condition, which should give conservative values. Table 1 lists the heat loads of the components and temperature rises of the coolant passing through the components. Components such as elbows and tees are not included since they will not have a significant effect on the overall heat load estimate. The average temperature rise on the fluid due to the detector heat load is roughly $1.68^{\circ} \mathrm{C}$ using the $640 \mathrm{~mW}$ chip power. This gives an overall fluid temperature rise of $2.24^{\circ} \mathrm{C}$.

\section{SYSTEM SAFETY}

The cooling channels are pressure tested to only $25 \mathrm{psig}$. Assuming the channel were to fail with any addition of pressure, this could be considered a burst pressure. A typical pressure vessel safety factor on a burst pressure is on the order of 4 to 5. Piping systems typically have a slightly lower safety factor. It was chosen to treat the cooling channels like pressure vessels. Therefore, the pressure rating is chosen to be $5 \mathrm{psig}$. There is good reason for this decision. The cooling channels have not been rigorously modeled and tested to know how good they really are. Therefore the uncertainty in the long term strength of the devices is quite high. Thus choosing a safety factor of 5 is justifiable considering the consequences of a failure. Protecting the cooling channels from exceeding 5 psig is not trivial.

Stopping the flow when the coolant is cold was examined. After cool-down, the density of the fluid increases so the level in the tank will drop slightly. When the flow is stopped, the coolant will warm and expand back to its original volume. If a section of pipe is isolated when cold, and allowed to warm-up, excessive pressure will buildup in the pipe section. To prevent this from happening, each section of pipe has a relief valve.

Over pressurization of the cooling channels needs to be considered in the design of the system. Since the expansion tank is at atmospheric pressure and upstream from the cooling channels, everything is fine during normal operation. If the flow downstream of 
the detector gets blocked, it's likely that the expansion tank will get filled and the pressure will begin to increase. The expansion tank is prevented from getting full through interlocks and also it is open to atmosphere. The cooling channels are safe from over pressurization. The pump is the only pressure source in the system. If the pump is starved of liquid, it cannot pressurize the supply piping. Devices down stream of the chiller are protected from over-pressurization with relief valves. Since we must control ethylene glycol spills, we must pipe the exhaust of the relief valves in the system and the expansion tank vents into a spill tank. This can be simply a 55 gallon barrel.

In addition to pressure safety, we need to make sure that we do not create mixed waste. Since the coolant will be exposed to radiation and the coolant is toxic, Fermilab Safety is requesting that we periodically take samples to send out for measuring the amount of tritium in the coolant. We are requested to change the coolant before we reach mixed waste levels. Based upon radiation estimates and calculations that were performed, we will not have to replace the coolant due to high levels of tritium. 